\title{
Associação entre risco cardiovascular e hipertensão arterial em professores universitários
}

CDD. 20.ed. 614.49

616.122

\author{
Osvaldo Costa MOREIRA* \\ Renata Aparecida Rodrigues de OLIVEIRA* \\ Flávio ANDRADE NETO* \\ William AMORIM ${ }^{*}$ \\ Cláudia Eliza Patrocínio OLIVEIRA* \\ Leonice Aparecida DOIMO* \\ Paulo Roberto dos Santos AMORIM ${ }^{*}$ \\ Mateus Camaroti LATERZA** \\ Walace David MONTEIRO**a \\ J oão Carlos Bouzas MARINS*
}

*Departamento de Educação Física, Universidade Federal de Viçosa.

**Faculdade de Educação Física e Desportos, Universidade Federal de Juiz de Fora.

***Departamento de Desportos Individuais, Universidade Estadual do Rio de Janeiro.

\section{Resumo}

A hipertensão arterial é uma das mais importantes causas modificáveis de morbi-mortalidade cardiovascular precoce na população adulta mundial, além de ser fator de risco independente para doenças cardiovasculares. Assim objetivou-se verificar a associação entre indicadores de risco cardiovascular e hipertensão arterial em professores universitários. Foram avaliados 145 professores da Universidade Federal de Viçosa (UFV), no ano de 2009, analisando-se as variáveis: sexo, idade, índice de massa corporal (IMC), relação cintura/quadril (RCQ), circunferência abdominal (CA), percentual de gordura $(\% \mathrm{G})$, colesterol total (CT), triacilglicerol (TG), glicose, pressão arterial sistólica (PAS) e diastólica (PAD) de repouso e consumo máximo de oxigênio $\left(\mathrm{VO}_{2 \text { maxx }}\right)$. Utilizou-se o teste ANOVA one way para comparação entre indivíduos normotensos e pré-hipertensos e hipertensos. Calculou-se a razão de chances de desenvolvimento de quadros de PA elevada entre os indivíduos que apresentavam níveis indicativos de risco cardiovascular. Todos os tratamentos possuíram nivel de significância de $p<0,05$. Os homens apresentaram valores significativamente maiores para idade, peso, estatura, IMC, RCQ, CA, PAS e PAD. Já as mulheres possuiam um $\% \mathrm{G}$ significativamente elevado. Os indivíduos com níveis pressóricos elevados apresentaram valores maiores para todas as variáveis, à exceção do $\mathrm{VO}_{2 \text { máx }}$ Observou-se associação os fatores de risco e elevação da PA somente para as variáveis: sexo, idade, IMC, CA, \%G e TG. Conclui-se que o gênero, a idade, o IMC, a circunferência abdominal, o percentual de gordura corporal e os triacilgliceróis foram considerados indicadores de risco cardiovascular por apresentarem associação com a hipertensão arterial na amostra de professores avaliados.

UnIteRMos: Epidemiologia; Doença crônica; Hipertensão arterial; Risco cardiovascular.

\section{Introdução}

A hipertensão arterial sistêmica (HAS) é uma das mais importantes causas modificáveis de morbimortalidade cardiovascular na população adulta mundial, além de ser fator de risco independente para doenças cardiovasculares (LEWINGTON, ClaRKE, Qizilbash, Peto \& Collins, 2002; Yusuf, Hawken, Ounpuu, Dans, Avezum, Lanas, McQueEn, Budaj,
Pais, Varigos \& Lisheng, 2004). No Brasil, a HAS possui elevada prevalência e está associada à consequências sociais e econômicas que a caracterizam como um grave problema de saúde pública (SOCIEDADE BRASILEIRA DE CARDIOLOGIA - SBC, 2007). Estima-se que, em 2006, 17 milhões de brasileiros apresentavam quadros de HAS, representando aproximadamente 
$35 \%$ do total de indivíduos adultos com mais de 40 anos (BRASIL, 2006).

Alguns estudos de caráter epidemiológico demonstram que a HAS, ou mesmo níveis elevados de pressão arterial (PA), aumentam o risco de doença vascular encefálica (YUSUF et al., 2004) doenças arteriais coronarianas (RUILOPE, 2009; ZDROJEWSKI, CHWOJNICKI, BANDOSZ, KONARSKI \& WYRZYKOWSKI, 2006), insuficiência cardíaca congestiva (HAYWOOD, Ford, Crow, Davis, Massie, Einhorn, Williard, \& Allhat Collaborative Research Group, 2009) e insuficiência renal crônica (RUILope, 2009; WAchtell, Papademetriou, Smith, Gerdts, Dahlöf, Engblom, Aurigemma, Bella, Ibsen, Rokkedal \& Devereux, 2004). Esta associação entre HAS e estes comprometimentos orgânicos torna essencial seu controle permanente, desde os sujeitos mais jovens, como uma ação preventiva fundamental.

A HAS associa-se ao histórico familiar de doença hipertensiva, bem como, a outros fatores de risco cardiovasculares modificáveis como sobrepeso e obesidade, sedentarismo, tabagismo, dislipidemias e diabetes mellitus (Barbosa, Silva, Santos, Monteiro Júnior, Barbosa, Barbosa, Figueiredo Neto, Soares, Nina \& Barbosa, 2008; Longo, Neves, Luciano \& Peres, 2009; SBC, 2007). Nesse sentido, alguns estudos têm investigado o nível de associação entre os fatores de risco cardiovascular de forma isolada (Ávila \& Marins, 1997; LesSA, Magalhāes, Araújo, Almeida Filho, Aquino \&
Oliveira, 2006; Castro, Moncau \& Marcopito, 2007; Souza, Costa, Nakamura, Mocheti, STeVANATO FiLHO \& Ovando, 2007) ou combinada com a HAS (Costa, Barcellos, Sclowitz, Sclowitz, Castanheira, Olinto, Menezes, Gigante, MaceDO \& Fuchs, 2007; Jardim, Gondim, Monego, Moreira, Vitorino, Souza \& Scala, 2007; Nunes Filho, Debastiani, Nunes \& Peres, 2007).

Um quadro de HAS também pode estar associado a determinadas funções laborais, como por exemplo, em servidores universitários (Moreira, OliveIra, Teodoro, Souza, Lizardo, Santos \& Marins, 2009), em funcionários de indústria de refrigerantes (Cassani, Nobre, Pazin Filho \& Schmidt, 2009) e em profissionais de saúde (SARNo \& Monteiro, 2007). Assim a partir da identificação dos fatores de risco cardiovascular que se associam com a HAS, em professores universitários, será possível elaborar e implantar estratégias educativas e protocolos de intervenção, visando o sucesso de ações preventivas e terapêuticas. Além disso, a partir do estudo dos fatores de risco cardiovascular que se associam com a HAS pode-se contribuir para direcionamento de açōes preventivas no sentido de torná-las mais eficientes e otimizar os recursos humanos e financeiros empregados em tais ações ações.

O presente trabalho teve como objetivo verificar o grau de associação entre diferentes indicadores de risco cardiovascular e hipertensão arterial em professores universitários.

\section{Métodos}

Trata-se de um estudo epidemiológico de delineamento transversal em população de professores universitários da Universidade Federal de Viçosa (UFV), ao longo do ano de 2009, independentemente do sexo, etnia e faixa etária.

O tamanho amostral foi calculado segundo a equação proposta por LWANGA e LEMESHOW (1991): n $=\mathrm{P} \times \mathrm{Q} /(\mathrm{E} / 1,96) 2 \mathrm{x}$, em que $\mathrm{n}=$ tamanho mínimo da amostra necessária; $\mathrm{P}=$ taxa máxima de prevalência; $\mathrm{Q}=100$ - P; $\mathrm{E}=$ margem de erro amostral tolerado. Como forma de comprovação probabilística foi necessária uma amostra de 138 professores, considerando o número total de professores da instituição (767 professores) e um erro padrão de 5\% com um intervalo de confiança de $98 \%$.

Após aprovação pelo Comitê de Etica para pesquisas com seres humanos da Universidade Federal de Viçosa (Of. Ref. No 009/2009/Comitê de Ética), todos os professores foram informados e convidados a participar deste estudo, empregando estratégias por carta convite, correio eletrônico pessoal, anúncio no site da instituição, além de campanhas em rádio e televisão. Mediante confirmação do interesse em participar e preenchimento do termo de consentimento livre e esclarecido, foi realizado um agendamento para avaliação individualizada.

Todas as avaliaçōes aconteceram no Laboratório de Performance Humana do Departamento de Educação Física da UFV, sendo executadas em uma sala climatizada (em torno de $22^{\circ} \mathrm{C}$ e $55 \%$ de umidade relativa do ar). Dois avaliadores experientes e treinados previamente para a rotina do estudo seguiram procedimento padrão, consistindo em: acompanhamento do avaliado para aferição da pressão 
arterial de repouso, antropometria, mensuração dos parâmetros sanguíneos e medida do componente cardiorrespiratório, com posterior elaboração de relatórios individuais para cada avaliado. Todos os testes foram realizados pelo mesmo avaliador, sendo que o segundo avaliador ficava incumbido de anotar os resultados obtidos. As variáveis antropométricas analisadas foram: idade, massa corporal, estatura, circunferência de cintura (CC), circunferência de quadril (CQ), circunferência abdominal (CA) e dobras cutâneas para cálculo do percentual de gordura $(\% \mathrm{G})$. Também foram investigados os níveis séricos de colesterol total (CT), triacilglicerol (TG) e glicose. Também foram aferidos o consumo máximo de oxigênio $\left(\mathrm{VO}_{2 \max }\right)$ e a pressão arterial, sistólica (PAS) e diastólica (PAD), de repouso.

A massa corporal foi medida em gramas por uma balança digital (Filizola, modelo Star 300/4, Brasil) calibrada, e a estatura, em centímetros, por um estadiômetro de parede (American Medical, modelo ES2020, Brasil), seguindo as recomendaçōes do International Society For the AdVANCEMENT of KinANTHROPOMETRY (2001). O índice de massa corporal (IMC) foi calculado e classificado segundo critérios preconizados pela OrganIZAÇÃo MUNDIAL DA SAÚdE - WHO (1998).

As CC, CQ e CA foram medidas em centímetro, utilizando-se fita métrica inelástica (American Medical, modelo SN4010, Brasil), graduada em milímetros. Os critérios para localização das medidas seguiram as recomendações do ISAK (2001). A classificação dos valores de relação cintura-quadril (RCQ) e dos valores CA obedeceu aos critérios propostos pela WHO (2000), que prevê valores diferentes para homens e mulheres.

Com a utilização de plicômetro (Cescorf, modelo Top Tec, Brasil) foram medidas as dobras cutâneas $(\mathrm{mm})$ triciptal, supra-ilíaca e coxa para mulheres e peitoral, abdominal e coxa para homens. A estimativa da densidade corporal foi realizado por meio da fórmula proposta por JACKSON e POLLOCK (1978). O resultado dessa estimativa foi empregado na equação de SIRI (1961) para estimar o \%G. Os procedimentos de medida das dobras cutâneas obedeceram aos critérios do ACSM (2006). A classificação do \%G foi feita com base nas normas de LOHMAN (1992).

A PAS e a PAD $(\mathrm{mmHg})$ foram aferidas por um esfigmomanômetro de coluna de mercúrio (Unilec) devidamente calibrado, com manguito padrão para adulto e estetoscópio. A realização das medidas e a classificação dos resultados foram efetuadas segundo critérios propostos pelo The Seventh Report of the Joint National Committee (CHOBANIAN, BaKris, Black, Cushman, Green, Izzo Junior, Jones, Materson, Oparil, Wright Junior \& Roccella, 2003) e endossados pela Sociedade Brasileira dE CARDiologia (2007).

As coletas de CT, TG e glicose foram realizadas por meio de punção digital, com o avaliado em repouso e jejum prévio de, pelo menos, quatro horas. Os equipamentos utilizados para a análise de sangue foram Accutrend PLUS (Roche, USA), para CT e TG e Accu-Chek Go (Roche, USA), para glicose. A classificação dos valores obtidos para CT e TG seguiu os critérios propostos pelo National Cholesterol Education Program do National Heart, Lung, AND Blood InstiTute (2002). Já a classificação dos valores obtidos para glicose seguiu a proposta $\mathrm{da}$ American Diabetes Association (2003). Todos os procedimentos de coleta e análise de sangue foram executados utilizando-se materiais descartáveis.

Para estimativa do $\mathrm{VO}_{2 \max }$ utilizou-se o teste de esteira submáximo de único estágio proposto por Ebbeling, Ward, Puleo, Widrick e Rippe (1991): início com três minutos de aquecimento com aumento progressivo de carga e inclinação, seguido por um estágio único de quatro minutos com carga e inclinaçãao constante, e finalizando com três minutos de recuperação com decréscimo progressivo de inclinação e velocidade. A opção por um protocolo submáximo de esteira deveu-se a características específicas apresentadas pela população, como idade avançada e atividade laboral sedentária, associadas ao desconhecimento da condição física desses indivíduos.

Todos os dados obtidos foram armazenados e analisados pelos programas estatísticos Epi Info ${ }^{\mathrm{TM}}$ versão 3.51 e Sigma Stat for Windows versão 2.03. A análise dos dados constituiu na exploração descritiva das variáveis estudadas e no cálculo da prevalência de HAS. Foi realizada a verificação da normalidade dos dados por meio do teste de Komolgorov-Smirnov. Utilizou-se o teste ANOVA one way, com "post hoc" de Tuckey para comparação entre os grupos de indivíduos normotensos, pré-hipertensos e hipertensos. Para verificação da razão de chances de desenvolvimento de quadros de PA elevada entre os indivíduos que apresentavam níveis indicativos de risco cardiovascular, em algum dos fatores analisados, realizou-se a "Odds Ratio", com intervalo de confiança de $95 \%$. Para todos os tratamentos adotou-se um nível de significância de $\mathrm{p}<0,05$. 


\section{Resultados}

Foram avaliados 145 professores universitários da UFV, com média de idade de 45,86 \pm 9,72 anos, o que correspondeu a $18,9 \%$ da população total de professores, sendo 103 homens $(71,03 \%)$ e 42 mulheres (28,97\%). A descrição dos valores médios para idade, peso, estatura, IMC, RCQ, CA, PAS, PAD, glicose sanguínea, CT, TG, $\mathrm{VO}_{2 \max }$ e $\% \mathrm{G}$ encontra-se na TABELA 1.

A PA média, tanto nos homens, quanto nas mulheres obteve classificação de PA normal (Chobanian et al., 2003; SBC, 2007). Entretanto, pode-se notar mais de $35 \%$ dos avaliados com níveis de PA elevados, como visto na TABELA 2.
A TABELA 3 mostra a comparação das médias de cada fator de risco entre aqueles indivíduos com PA normal e aqueles que apresentaram alguma elevação da PA (pré-hipertensão e hipertensão estágio 1). Observa-se que os indivíduos com elevação dos níveis pressóricos apresentaram todos os indicadores de risco cardiovascular mais elevados, quando comparados à seus congêneres normotensos. Entretanto, somente contatou-se associação estatisticamente significante, por meio da OR, entre a elevação da PA e os fatores de risco sexo, idade, IMC, CA, \%G e TG, como pode ser visto na TABELA 4.

TABELA 1 - Caracterização da amostra segundo o gênero.

\begin{tabular}{lccc}
\hline \multicolumn{1}{c}{ Variável } & $\begin{array}{c}\text { Masculino }(\mathbf{n}=\mathbf{~ 1 0 3}) \\
\text { Média } \pm \text { DP }\end{array}$ & $\begin{array}{c}\text { Feminino }(\mathbf{n}=\mathbf{4 2}) \\
\text { Média } \pm \text { DP }\end{array}$ & P valor \\
\hline Idade $(\mathrm{anos})$ & $46,90 \pm 9,82$ & $43,27 \pm 9,09$ & 0,042 \\
Peso $(\mathrm{kg})$ & $77,56 \pm 11,97$ & $62,10 \pm 9,89$ & $<0,001$ \\
Estatura $(\mathrm{m})$ & $1,74 \pm 0,07$ & $1,61 \pm 0,06$ & $<0,001$ \\
$\mathrm{IMC}\left(\mathrm{kg} / \mathrm{m}^{2}\right)$ & $25,57 \pm 3,54$ & $24,11 \pm 3,65$ & 0,027 \\
$\% \mathrm{G}$ & $22,58 \pm 6,05$ & $31,37 \pm 6,83$ & $<0,001$ \\
$\mathrm{RCQ}$ & $0,89 \pm 0,06$ & $0,78 \pm 0,06$ & $<0,001$ \\
$\mathrm{CA}(\mathrm{cm})$ & $92,51 \pm 10,01$ & $82,35 \pm 9,08$ & $<0,001$ \\
$\mathrm{Glicose}(\mathrm{mg} / \mathrm{dl})$ & $86,19 \pm 11,44$ & $85,71 \pm 13,59$ & 0,829 \\
$\mathrm{CT}(\mathrm{mg} / \mathrm{dl})$ & $183,15 \pm 28,86$ & $183,26 \pm 34,39$ & 0,882 \\
$\mathrm{TG}(\mathrm{mg} / \mathrm{dl})$ & $159,20 \pm 72,78$ & $142,18 \pm 66,46$ & 0,217 \\
VO ${ }_{2 \text { máx }} \mathrm{ml}(\mathrm{kg} \cdot \mathrm{min})^{-1}$ & $43,34 \pm 6,12$ & $35,85 \pm 5,98$ & $<0,001$ \\
PAS $(\mathrm{mmHg})$ & $118,79 \pm 10,01$ & $111,67 \pm 12,28$ & $<0,001$ \\
PAD $(\mathrm{mmHg})$ & $78,64 \pm 7,80$ & $74,88 \pm 8,94$ & 0,013 \\
\hline
\end{tabular}

TABELA 2 - Distribuição dos indivíduos de acordo com a classificação da pressão arterial.

\begin{tabular}{llllccccc} 
& Classificação & \multicolumn{2}{c}{ Normal } & \multicolumn{2}{c}{ Pré-hipertensão } & \multicolumn{2}{c}{ Hipertensão estágio 1 } & \multicolumn{2}{c}{ Total } \\
*Nãoforam encontrados & \multicolumn{1}{c}{ JNC7 } & $\mathbf{n}$ & $\mathbf{\%}$ & $\mathbf{n}$ & $\mathbf{\%}$ & $\mathbf{n}$ & $\mathbf{\%}$ & $\mathbf{n}$ \\
\cline { 2 - 10 } casos de hipertensão & Masculino & 60 & 58,25 & 22 & 21,36 & 21 & 20,39 & 103 \\
estágio 2. & Feminino & 33 & 78,57 & 06 & 14,29 & 03 & 7,14 & 42 \\
& Total & 93 & 64,14 & 28 & 19,31 & 24 & 16,55 & 145 \\
\hline
\end{tabular}


TABELA 3 - Comparação dos valores médios para cada fator derisco cardiovascular entreindivíduos normotensos, pré-hipertensos e hipertensos.

\begin{tabular}{lccc}
\hline \multicolumn{1}{c}{ Fator de risco } & Normotensos $(\mathbf{n}=\mathbf{9 3})$ & Pré-hipertensos $(\mathbf{n}=\mathbf{2 8})$ & Hipertensos $(\mathbf{n}=\mathbf{2 4})$ \\
\hline Idade $(\mathrm{anos})$ & $43,76 \pm 9,04$ & $42,36 \pm 7,33$ & $58,04 \pm 3,77^{* \dagger}$ \\
$\mathrm{IMC}\left(\mathrm{kg} / \mathrm{m}^{2}\right)$ & $24,09 \pm 2,73$ & $26,36 \pm 3,21^{*}$ & $27,82 \pm 5,14^{*}$ \\
$\mathrm{RCQ}$ & $0,84 \pm 0,07$ & $0,86 \pm 0,07$ & $0,91 \pm 0,07^{*}$ \\
$\mathrm{CA}(\mathrm{cm})$ & $85,96 \pm 8,76$ & $92,89 \pm 8,64^{*}$ & $99,69 \pm 12,58^{* \dagger}$ \\
$\% \mathrm{G}$ & $24,15 \pm 6,89$ & $26,33 \pm 8,66$ & $27,54 \pm 7,51$ \\
$\mathrm{VO}_{2 \text { máx }} \mathrm{ml}(\mathrm{kg} \cdot \mathrm{min})^{-1}$ & $41,45 \pm 7,1$ & $44,02 \pm 7,19$ & $36,73 \pm 3,07^{* \dagger}$ \\
$\mathrm{Glicose}(\mathrm{mg} / \mathrm{dl})$ & $83,83 \pm 8,25$ & $86,25 \pm 6,68$ & $94,46 \pm 22,16^{*} \dagger$ \\
$\mathrm{CT}(\mathrm{mg} / \mathrm{dl})$ & $164,22 \pm 61,81$ & $186,61 \pm 26,92$ & $183,11 \pm 30,83$ \\
$\mathrm{TG}(\mathrm{mg} / \mathrm{dl})$ & $122,07 \pm 73,27$ & $169,71 \pm 77,4$ & $189,3 \pm 88,91^{*}$ \\
\hline
\end{tabular}

TABELA 4 - Razão de chance ("Odds Ratio" - OR e intervalo de confiança de 95\%) de elevação da pressão arterial segundo presença de fatores de risco cardiovascular em professores da UFV.

\begin{tabular}{|c|c|c|c|}
\hline Variáveis & Fator de risco (n) & Razão de prevalência (IC 95\%) & P-valor \\
\hline Sexo & Masculino (103) & $2,63(1,07-6,61)$ & 0,02 \\
\hline Idade & $>40$ anos $(104)$ & $2,51(1,02-6,32)$ & 0,03 \\
\hline IMC & $>24,49 \mathrm{~kg} / \mathrm{m}^{2}(81)$ & $2,75(1,26-6,06)$ & $<0,01$ \\
\hline RCQ & $\delta \geq 0,95 / q \geq 0,85(25)$ & $2,25(0,87-5,87)$ & 0,06 \\
\hline CA & $\delta \geq 94 / q \geq 80$ (68) & $2,53(1,19-5,41)$ & $<0,01$ \\
\hline$\% G$ & $\delta \geq 25 / q \geq 32(54)$ & $3,01(1,40-6,51)$ & $<0,01$ \\
\hline $\mathrm{VO}_{2 \text { máx }}$ & Inadequado* (16) & $0,56(0,14-2,03)$ & 0,34 \\
\hline Glicose & $\geq 100 \mathrm{mg} / \mathrm{dl}(07)$ & $4,84(0,79-37,61)$ & 0,11 \\
\hline CT & $>200 \mathrm{mg} / \mathrm{dl}(36)$ & $0,97(0,39-2,37)$ & 0,93 \\
\hline TG & $\geq 150 \mathrm{mg} / \mathrm{dl}(55)$ & $3,93(1,77-8,79)$ & $<0,01$ \\
\hline
\end{tabular}

*Segundo critérios propostos por COOPER (1982);

§̧: gênero masculino; †: gênero feminino.

\section{Discussão}

Para critério de classificação da PA, adotou-se um valor tensional de PAS maior ou igual a $140 \mathrm{mmHg}$ e/ou PAD maior ou igual a $90 \mathrm{mmHg}$, como sendo indicativo de HAS (CHOBANIAN et al., 2003; SBC, 2007), onde verificou-se uma prevalência de HAS de $16,55 \%$ na população estudada, sendo que $20,39 \%$ nos homens e $7,14 \%$ nas mulheres.

Utilizando-se esse mesmo critério, foram encontradas prevalências de HAS em capitais brasileiras que variavam entre 15,1\% em Palmas-TO e 24,9\% em Recife-PE (Brasil, 2007). Entre os homens, segundo dados do Ministério da Saúde (Brasil, 2007), as maiores prevalências foram observadas em Recife-PE (22,5\%), Belo Horizonte (22,7\%) e Vitória-ES $(23,1 \%)$ e as menores em FlorianópolisSC (14,9\%), Palmas-TO (14,9\%) e Brasília-DF
(15,5\%), o que demonstra que os professores do sexo masculino possuem prevalência de HAS semelhante à realidade nacional.

Contudo, as mulheres demonstraram prevalência bem abaixo dos valores encontrados nas capitais brasileiras, onde as maiores frequências foram observadas em Recife-PE (26,8\%), Salvador-BA $(27,3 \%)$ e Rio de Janeiro-RJ $(28,0 \%)$ e as menores em Palmas-TO (15,3\%), Teresina-PI $(18,4 \%)$ e Manaus-AM (19,2\%) (BrAsIL, 2007), indicando que algumas características do grupo feminino, como o nível de escolaridade (JARDIM et al., 2007), maior prática de autocuidados com a saúde e hábitos alimentares saudáveis (BARBOSA et al., 2008), podem estar atuando como fator de proteção, contra o desenvolvimento de quadros hipertensivos.
${ }^{*} \mathrm{P}$-valor $<0,05$ em comparação com grupo de normotensos; † P-valor < 0,05 em comparação com grupo de pré-hipertensos.

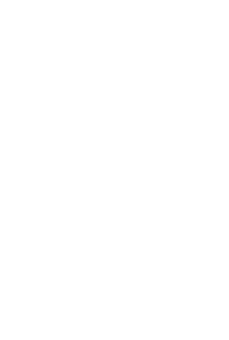

Rev. bras. Educ. Fís. Esporte, São Paulo, v.25, n.3, p.397-406, jul./ set. 2011 • 401 
Apesar do fator estresse não ter sido avaliado no presente estudo, pode-se considerar a hipótese de que, as mulheres de Viçosa não enfrentam o estresse típico das grandes cidades visto que trabalham em uma Universidade do interior do Estado, sendo esse, um aspecto que pode estar relacionado com uma melhor qualidade de vida das mesmas. Além disso, em pesquisa realizada por nosso grupo, em amostra de professores da mesma instituição, constatou-se que as mulheres apresentam níveis mais elevados de atividade física, quando comparadas aos homens (dados não publicados), o que pode estar impactando positivamente os níveis pressóricos, diminuindo assim, a prevalência de HAS nas professoras.

Considerando-se estudos que avaliaram a prevalência de HAS em cidades do interior do Brasil, utilizando-se os critérios supracitados, temos: Pelotas-RS (23,6\%) (CostA et al., 2007); Lages-SC (33,7\%) (LonGO et al., 2009); Formiga-MG (32,7\%) (Castro, Moncau \& Marcopito, 2007); e Virgem das Graças-MG (47\%) (Pimenta, KaC, GaZZInelli, CorrêA-Oliveira \& Velásquez-Meléndez, 2008).

A constatação de menor prevalência de HAS nos professores da UFV, quando comparados a outros estudos brasileiros pode ser explicada pela realização de iniciativas na instituição, no sentido de determinar e prevenir a prevalência de HAS, tais como realização de palestras sobre os riscos associados à HAS e sobre os benefícios dos exercícios físicos regulares, dias temáticos sobe a PA, bem como estudos para verificar a prevalência de HAS na instituição. Estes têm obtido valores de prevalência de $65,9 \%$ em servidores (Ávila \& Marins, 1997), 23,2\% em professores e técnicos administrativos (Moreira, Oliveira \& Marins, 2008), e 20,3\% em técnicos administrativos (Moreira et al., 2009). Tais valores demonstram que existe uma tendência de diminuição da prevalência de HAS entre os servidores da UFV, que pode ter sido ocasionada por ações de prevenção e tratamento dessa doença no campus da instituição.

Além disso, a atuação profissional, como professor universitário, o que exigem um nível de escolaridade elevado (mestrado ou doutorado), pode estar influenciando positivamente na redução da HAS nesse extrato populacional (JARDIM et al., 2007). Adicionalmente, a produção de conhecimento efetuada por parte dos cursos do Centro de Ciências Biológicas e da Saúde, é outro fator que também pode estar contribuindo para a conscientização dos professores e adoção de medidas que impliquem em hábitos de vida mais saudáveis e que, consequentemente, possam provocar redução de seus níveis tensionais.
O gênero associou-se positivamente à elevação da PA, sendo que os homens apresentaram níveis tensionais mais elevados que as mulheres, tanto para PAS, quanto para PAD. Além disso, os homens possuíam 2,63 vezes mais chances de apresentarem elevação da PA, que as mulheres da presente amostra. Esse resultado converge com os relatados por BARBOSA et al. (2008), SouZA et al. (2007) e JARDiM et al. (2007). Entretanto, existe uma divergência entre os resultados do presente estudo e os encontrados por Castro, Moncau e Marcopito (2007), Costa et al. (2007), Brasil (2007) e LONGO et al. (2009).

Associaçōes positivas entre HAS e idade são relatadas por diversos estudos (BARBOSA et al., 2008; CASTRO, Moncau \& Marcopito, 2007; Costa et al., 2007; JARDIM et al., 2007; LONGO et al., 2009; PIMENTA et al., 2008; SouzA et al., 2007). De forma análoga, a idade associou-se positivamente ao aumento pressórico, sendo que o grupo de indivíduos com mais de 40 anos de idade possuem 2,51 vezes mais chances de ter elevação da PA, que seus congêneres com menos de 40 anos. Nos professores da UFV, a idade também foi determinante para o risco de hipertensão. Considerando que a idade média do grupo foi de 45,86 $\pm 9,72$ anos, indica que, caso não sejam tomadas medidas preventivas, poderá ocorrer aumento dos valores tensionais.

O processo de envelhecimento desencadeia alteraçôes fisiológicas próprias, que tornam os indivíduos mais propensos ao desenvolvimento de HAS, sendo essa a principal doença crônica na população de idosos (SouZA et al., 2007). O estudo de Framingham (Franklin, Khan, Wong, Larson \& LeVY, 1999) constatou que a PAS aumenta continuamente com a idade. Assim, ações que visem combater o aumento dos níveis tensionais devem focar outros fatores associados à HAS, que sejam passíveis de alteração, minimizando a possibilidade de interação desses fatores com a idade.

Foi verificada associação positiva entre a elevação da PA e indicadores antropométricos, tais como IMC, CA e \%G. Indivíduos com IMC maior que 24,49 $\mathrm{kg} / \mathrm{m}^{2}$ apresentavam 2,75 vezes mais chances de ter elevação da $\mathrm{PA}$, que seus congêneres com valores menores ou iguais à esse índice. Esse achado encontrase em consonância com alguns estudos nacionais (BARBosa et al., 2008; Costa et al., 2007; JardiM et al., 2007; Longo et al., 2009; SouZA et al., 2007). Níveis elevados de IMC, CA e \%G estão associados a hábitos sedentários e ao desequilíbrio nutricional (Gelbrich, Blüher, Reich, Müller \& Kiess, 2008). Desta forma, uma intervençáo através de aumento dos níveis de atividade física diária, prática de exercícios 
físicos regulares e controle da ingestão calórica podem impactar positivamente esses fatores, diminuindo sua participação na gênese da HAS.

Os indivíduos com CA aumentada (maior ou igual a $94 \mathrm{~cm}$ para homens e maior ou igual a $80 \mathrm{~cm}$ para mulheres) possuíam 2,53 vezes mais chances de apresentarem elevação da PA. Semelhantemente, um estudo que utilizou os mesmos critérios para classificação da CA com uma população adulta residente na região urbana do município de Formiga-MG, também constatou que os sujeitos com CA aumentada tinham 3,05 vezes mais chances de apresentarem níveis pressóricos elevados quando comparados àqueles com CA normal (CASTro, MonCAU \& Marcopito, 2007). Esta informação é importante tendo em vista que medidas antropométricas são de execução extremamente simples, podendo ser amplamente divulgadas à população, visando a automonitoração, como forma de estratificação de risco.

Adicionalmente, indivíduos com $\% \mathrm{G}$ excessivo (maior ou igual a $25 \%$ para homens e maior ou igual a $32 \%$ para mulheres) tinham 3,01 vezes mais chances de ter elevação da PA, que os indivíduos com \%G normal. Até onde se pode verificar na literatura nacional (BARBosa et al., 2008; CASTRO, Moncau \& Marcopito, 2007; Longo et al., 2009; Moreira et al., 2009; Pimenta et al., 2008), nenhum estudo avaliou o $\% \mathrm{G}$ como fator de risco para HAS, nem tampouco o associou à elevação dos níveis pressóricos, constituindo-se assim, uma barreira para comparação dos resultados.

A prevalência da HAS sofre influência do excesso de peso, e sua gravidade parece aumentar diretamente com a quantidade de gordura corporal e com o padrão de distribuição predominantemente visceral (GaLVÃo \& Kohlmann, 2002), o que corrobora com os achados do presente estudo. A associação entre os indicadores antropométricos de excesso de peso e a ocorrência de elevação dos níveis tensionais indica a importância e urgência da realização de ações capazes de atuar sobre os fatores de risco, no intuito de interferir decisivamente sobre a prevalência de hipertensão arterial nesse grupo populacional.

Em relação aos parâmetros sanguíneos analisados, o único que apresentou associação positiva com a elevação pressórica foi o TG, sendo que indivíduos com níveis sanguíneos de TG iguais ou maiores que $150 \mathrm{mg} / \mathrm{dl}$, possuíam 3,93 vezes mais chances de apresentarem elevação da PA, que seus congêneres com níveis mais baixos. De forma similar, PIMENTA et al. (2008), em estudo realizado na população adulta de Virgem das Graças-MG, encontraram associação positiva entre os níveis de TG e PA elevada, sendo que indivíduos com TG elevado tinham 6,04 vezes mais chances de ter elevação da PA do que aqueles com níveis de TG normais.

Os dados de TG reforçam a importância da adoção de medidas de prevenção e controle de fatores de risco, visto que, além de atuar sobre a gênese da HAS, existem indícios de que níveis elevados de TG representam o fator de risco mais significante associado a doenças cardiovasculares (COUGHLAN \& Sorrentino, 2000).

$\mathrm{O}$ presente estudo apresentou algumas limitações por não analisar outras variáveis, que podem associar-se à HAS, como os hábitos alimentares de ingestão de sódio e gordura. Outra limitação em estudos transversais é a possibilidade de ocorrer causalidade reversa, o que poderia alterar a interpretação de resultados (BARBOSA et al., 2008). Entretanto, nota-se uma convergência dos resultados obtidos com os dados relatados por vários estudos nacionais.

\section{Conclusão}

Foi verificado que os indicadores de risco cardiovascular que apresentaram associação com a hipertensão arterial em professores da UFV foram o gênero, a idade, o IMC, a circunferência abdominal, o percentual de gordura corporal e os triacilgliceróis.
Essa constatação denota a importância da inserção de políticas de promoção de saúde, pautadas em ações específicas que atuem sobre os indicadores de risco cardiovascular que se mostraram associados à HAS. 


\begin{abstract}
Association between cardiovascular risk and hypertension in universities professors

Hypertension is one of the most important modifiable causes of precocious cardiovascular morbidmortality in the adult population worldwide, and it's an independent risk factor for cardiovascular disease. Thus we aimed to investigate the association between cardiovascular risk and blood pressure on universities professors. 145 professors from Universidade Federal de Viçosa (UFV) were evaluated, in 2009, analyzing the variables: gender, age, body mass index (BMI), waist / hip ratio (WHR), abdominal circumference (AC), percent body fat (\% BF), total cholesterol (TC), triglyceride (TG), glucose, systolic blood pressure (SBP) and diastolic (DBP) at rest and maximal oxygen uptake $\left(\mathrm{VO}_{2 \max }\right)$. We used ANOVA one way test for comparison between normotensive and prehypertensive and hypertensive individuals. It was calculated the odds ratio of high BP expansion among individuals who had indicative levels of cardiovascular risk. All treatments possessed a significance level of $p<0.05$. Men showed significantly higher values for age, weight, height, BMI, WHR, AC, SBP and DBP. The women have had significantly higher $\% \mathrm{BF}$. Individuals with high blood pressure had higher values for all variables, except for $\mathrm{VO}_{2 \text { max }}$. Association between risk factors and blood pressure increase was only observed to gender, age, BMI, $\% \mathrm{BF}$ and $\mathrm{BT}$. It is concluded that gender, age, BMI, abdominal circumference, percentage body fat and triglycerides were considered risk factors for cardiovascular disease associated with hypertension in the sample of professors evaluated.
\end{abstract}

Uniterms: Epidemiology; Chronic disease; Hypertension; Cardiovascular risk.

\title{
Referências
}

AMERICAN COLLEGE OF SPORTS MEDICINE. Manual do ACSM para avaliação da aptidão física relacionada à saúde. Rio de Janeiro: Guanabara Koogan, 2006.

AMERICAN DIABETES ASSOCIATION. The Expert Comitee on the Diagnosis and Classification of Diabetes Mellitus. Follow-up report on the diagnosis of diabetes mellitus. Diabetes Care, Alexandria, v.26, p.3160-7, 2003.

ÁVILA, A.A.; MARINS, J.C.B. Levantamento epidemiológico dos valores de pressão arterial na comunidade universitária da Universidade Federal de Viçosa. Revista da Sociedade de Cardiologia do Estado de São Paulo, São Paulo, v.7, n.2, p.22-2, 1997. BARBOSA, J.B.; SILVA, A.A.M.; SANTOS, A.M.; MONTEIRO JÚNIOR, F.C.; BARBOSA, M.M.; BARBOSA, M.M.; FIGUEIREDO NETO, J.A.; SOARES, N.J.S.; NINA, V.J.S.; BARBOSA, J.N. Prevalência da hipertensão arterial em adultos e fatores associados em São Luís - MA. Arquivos Brasileiros de Cardiologia, São Paulo, v.91, n.4, p.260-6, 2008. BRASIL. Ministério da Saúde. Secretaria de Atenção à Saúde. Departamento de Atenção Básica. Hipertensão arterial sistêmica para o Sistema Único de Saúde. Brasília: MS, 2006. (Cadernos de Atenção Básica; 16/ Série A. Normas e Manuais Técnicos).

BRASIL. Ministério da Saúde. Vigitel Brasil 2006: vigilância de fatores de risco e proteção para doenças crônicas por inquérito telefônico. Brasília: MS, 2007. Disponível em: <http://bvsms.saude.gov.br/bvs/publicacoes/vigitel_2006.pdf>. Acesso em: 12 jan. 10

CASSANI, R.S.L.; NOBRE, F.; PAZIN FILHO, A.; SCHMIDT, A. Prevalência de fatores de risco cardiovascular em trabalhadores de uma indústria brasileira. Arquivos Brasileiros de Cardiologia, São Paulo, v.92, n.1, p.16-22, 2009.

CASTRO, R.A.A.; MONCAU, J.E.C.; MARCOPITO, L.F. Prevalência de hipertensão arterial sistêmica na Cidade de Formiga, MG. Arquivos Brasileiros de Cardiologia, São Paulo, v.88, n.3, p.334-9, 2007.

CHOBANIAN, A.V.; BAKRIS, G.L.; BLACK, H.R.; CUSHMAN, W.C.; GREEN, L.A.; IZZO JUNIOR, J.L.; JONES, D.W.; MATERSON, B.J.; OPARIL, S.; WRIGHT JUNIOR, J.T.; ROCCELLA, E.J. Joint National Committee on Prevention, Detection, Evaluation, and Treatment of High Blood Pressure. National Heart, Lung, and Blood Institute; National High Blood Pressure Education Program Coordinating Committee. Seventh report of the Joint National Committee on Prevention, Detection, Evaluation, and Treatment of High Blood Pressure. Hypertension, Dallas, v.42, p.1206-52, 2003. COOPER, K.N. The aerobics program for total well-being. Toronto: Bantam Books, 1982. 
COSTA, J.S.D.; BARCELLOS, F.C.; SCLOWITZ, M.L.; SCLOWITZ, I.K.T.; CASTANHEIRA, M.; OLINTO, M.T.A.; MENEZES, A.M.B.; GIGANTE, D.P.; MACEDO, S.; FUCHS, S.C. Prevalência de hipertensão arterial em adultos e fatores associados: um estudo de base populacional urbana em Pelotas, Rio Grande do Sul, Brasil. Arquivos Brasileiros de Cardiologia, São Paulo, v.88, n.1, p.59-65, 2007.

COUGHLAN, B.J.; SORRENTINO, M.J. Does hypertriglyceridemia increase risk for CAD? Growing evidence suggests it plays a role. Postgraduate Medicine, Berwyn, v.108, p.77-84, 2000.

EBBELING, C.B.; WARD, A.; PULEO, E.M.; WIDRICK, J.; RIPPE, J.M. Development of a single-stage submaximal treadmill walking test. Medicine and Science Sports and Exercise, Madison, v.23, n.8, p.966-73, 1991.

FRANKLIN, S.S.; KHAN, A.S.; WONG, N.D.; LARSON, M.G.; LEVY, D. Is pulse pressure useful in predicting risk for coronary heart disease? The Framingham Heart Study. Circulation, Dallas, v.100, n.4, p.354-60, 1999.

GALVÃO, R.; KOHLMANN, O.J. Hipertensão arterial no paciente obeso. Revista Brasileira de Hipertensão, Ribeirão Preto, v.9, n.2, p.262-7, 2002.

GELBRICH, G.; BLÜHER, S.; REICH, A.; MÜLLER, G.; KIESS, W. Prevalence of obesity and elevated blood pressure as well as onset of puberty in German school children attending different educational tracks. Hormone Research, Basel, v.70, n.6, p.340-8, 2008.

HAYWOOD, L.J.; FORD, C.E.; CROW, R.S.; DAVIS, B.R.; MASSIE, B.M.; EINHORN, P.T.; WILLIARD, A.; ALLHAT Collaborative Research Group. Atrial fibrillation at baseline and during follow-up in ALLHAT (Antihypertensive and Lipid-Lowering Treatment to Prevent Heart Attack Trial). Journal of the American College of Cardiology, New York, v.54, n.22, p.2023-31, 2009.

INTERNATIONAL SOCIETY FOR THE ADVANCEMENT OF KINANTHROPOMETRY (ISAK). International standards for anthropometric assessment. Adelaid: National Library of Australia , 2001.

JACKSON, A.S.; POLLOCK, M.L. Generalized equations for predicting body density of men. British Journal of Nutrition, Wallington, v.40, p.497-504, 1978.

JARDIM, P.C.B.V.; GONDIM, M.R.P.; MONEGO, E.T.; MOREIRA, H.G.; VITORINO, P.V.O.; SOUZA, W.K.S.B.; SCALA, L.C.N. Hipertensão arterial e alguns fatores de risco em uma capital brasileira. Arquivos Brasileiros de Cardiologia, São Paulo, v.88, n.4, p.452-7, 2007.

LESSA, I.; MAGALHĀES, L.; ARAÚJO, M.J.; ALMEIDA FILHO, N.; AQUINO, E.; OLIVEIRA, M.M.C. Hipertensão arterial na população adulta de Salvador (BA) - Brasil. Arquivos Brasileiros de Cardiologia, São Paulo, v.87, n.6, p.747-56, 2006. LEWINGTON, S.; CLARKE, R.; QIZILBASH, N.; PETO, R.; COLLINS, R.; for the Prospective Studies Collaboration. Age-specific relevance of usual blood pressure to vascular mortality: a meta-analysis of individual data for one million adults in 61 prospective studies. Lancet, New York, v.360, p.1903-13, 2002.

LOHMAN, T.G. Advances in body composition assessment. Champaign: Human Kinetics, 1992. Monograph Number 3. LONGO, G.Z.; NEVES, J.; LUCIANO, V.M.; PERES, M.A. Prevalência de níveis pressóricos elevados e fatores associados em adultos de Lages/SC. Arquivos Brasileiros de Cardiologia, São Paulo, v.93, n.3, p.387-94, 2009.

LWANGA, S.K.; LEMESHOW, S. Sample size determination in health studies: a practical manual. Geneve: World Health Organization, 1991.

MOREIRA, O.C.; OLIVEIRA, C.E.P.; MARINS, J.C.B. Diagnóstico primário de fatores de risco coronarianos em professores e técnicos administrativos do Centro de Ciências Agrárias da UFV. Lecturas: Educación Física y Deportes Buenos Aires, v.13, p.1, 2008.

MOREIRA, O.C.; OLIVEIRA, C.E.P.; TEODORO, B.G.; SOUZA, G.C.; LIZARDO, F.B.; SANTOS, L.A.; MARINS, J.C.B. Fatores de risco de doença cardiovascular em técnicos administrativos da Universidade Federal de Viçosa. Bioscience Journal, Uberlândia, v.25, n.5, p.133-40, 2009.

NATIONAL HEART, LUNG, AND BLOOD INSTITUTE. Third Report of the National Cholesterol Education Program (NCEP). Expert panel on detection, evaluation, and treatment of high blood cholesterol in adults (Adult Treatment Panel III): final report. Bethesda: National Heart, Lung, and Blood Institute, 2002. NIH Publication n. 02-5215.

NUNES FILHO, J.R.; DEBASTIANI, D.; NUNES, A.D.; PERES, K.G. Prevalência de fatores de risco cardiovascular em adultos de Luzerna, Santa Catarina, 2006. Arquivos Brasileiros de Cardiologia, São Paulo, v.89, n.5, p.319-24, 2007. PIMENTA, A.M.; KAC, G.; GAZZINELLI, A.; CORRÊA-OLIVEIRA, R.; VELÁSQUEZ-MELÉNDEZ, G. Associação entre obesidade central, triglicerídeos e hipertensão arterial em uma área rural do Brasil. Arquivos Brasileiros de Cardiologia, São Paulo, v.90, n.6, p.419-25, 2008.

RUILOPE, L.M. Arterial hypertension greatly contributes to the development and evolution of cardiovascular and renal diseases (Editorial). Journal of Hypertension, London, v.27, p.S1, 2009. Supplement 3. 
SARNO, F; MONTEIRO, C.A. Importância relativa do índice de massa corporal e da circunferência abdominal na predição da hipertensão arterial. Revista de Saúde Pública, São Paulo, v.41, n.5, p.788-96, 2007.

SIRI, W.E. Body composition from fluid spaces and density: analyses of methods. In: BROZEK, J.; HENSCHEL, A. (Eds.). Techniques for measuring body composition. Washington: National Academy of Science, 1961.

SOCIEDADE BRASILEIRA DE CARDIOLOGIA. V Diretrizes brasileiras de hipertensão. Arquivos Brasileiros de Cardiologia, São Paulo, v.89, n.3, p.e24-e79, 2007.

SOUZA, A.R.A.; COSTA, A.; NAKAMURA, D.; MOCHETI, L.N.; STEVANATO FILHO, P.R.; OVANDO, L.A. Um estudo sobre hipertensão arterial sistêmica na Cidade de Campo Grande, MS. Arquivos Brasileiros de Cardiologia, São Paulo, v.88, n.4, p.441-6, 2007.

WACHTELL, K.; PAPADEMETRIOU, V.; SMITH, G.; GERDTS, E.; DAHLÖF, B.; ENGBLOM, E.; AURIGEMMA, G.P.; BELLA, J.N.; IBSEN, H.; ROKKEDAL, J.; DEVEREUX, R.B. Relation of impaired left ventricular filling to systolic midwall mechanics in hypertensive patients with normal left ventricular systolic chamber function: The Losartan Intervention for Endpoint Reduction in Hypertension (LIFE) study. American Heart Journal, St. Louis, v.148, p.538-44, 2004. WORLD HEALTH ORGANIZATION. National Institutes of Health. Clinical guidelines on the identification, evaluation, and treatment of overweight and obesity in adults-the evidence report. National Institutes of Health. Obesity Research, Silver Spring, v.6, p.51-209, 1998. Supplement 2.

. Obesity: preventing and managing the global epidemic. Report of a WHO consultation. World Health Organization Technical Reports Series, Geneva, n.894, p.i-xii,1-253, 2000.

YUSUF, S.; HAWKEN, S.; OUNPUU, S.; DANS, T.; AVEZUM, A.; LANAS, F.; McQUEEN, M.; BUDAJ, A.; PAIS, P.; VARIGOS, J.; LISHENG, L. Effect of potentially modifiable risk factors associated with myocardial infarction in 52 countries (the INTERHEART study): case-control study. Lancet, New York, v.364, p.937-52, 2004.

ZDROJEWSKI, T.; CHWOJNICKI, K.; BANDOSZ, P.; KONARSKI, R.; WYRZYKOWSKI, B. Distribution of Creactive protein and its relation to arterial hypertension in a country representing a high-risk region for cardiovascular diseases. Blood Pressure, London, v.15, n.1, p.20-6, 2006.

\begin{tabular}{r|l} 
ENDEREÇO & \\
Osvaldo Costa Moreira & \\
R. Magalhães Pinto, 25 & Recebido para publicação: 30/09/2010 \\
35690-000 - Florestal - MG - BRASIL & 1a. Revisão: 27/04/2011 \\
e-mail: osvaldo.moreira@ufv.br & 2a. Revisão: 27/06/2011 \\
& 3a. Revisão: 15/07/2011 \\
& Aceito: 04/08/2011
\end{tabular}

406 • Rev. bras. Educ. Fís. Esporte, São Paulo, v.25, n.3, p.397-406, jul./ set. 2011 